\title{
Les projets pédagogiques entrepreneuriaux au Nouveau-Brunswick : Conceptualisation d'une approche collaborative structurante
}

\author{
Clara Jates ${ }^{1}$, Place aux compétences \\ Lise Gallant, Place aux compétences \\ Jalila Jbilou, Université de Moncton \\ Nicolas Washburn, Université du Québec à Montréal \\ Alain Poitras, Place aux compétences \\ Jean-Louis Caron, Place aux compétences
}

\section{INTRODUCTION}

Les jeunes sont dorénavant appelés à développer leur capacité à s'entreprendre de même qu'à acquérir un ensemble de compétences afin de mieux se préparer aux études, au travail ou à la vie en général.

Apprendre à «s'entreprendre»: une nouvelle nécessité pour répondre aux besoins de demain! C'est ainsi que les jeunes sont de plus en plus encouragés à s'impliquer et à s'engager activement dans des activités étroitement reliées au monde du travail ${ }^{2}$. Le marché du travail est cependant en constante évolution ce qui rend sa nature changeante et imprévisible. Ainsi, plus que jamais, les individus sont appelés à s'adapter à cette nouvelle réalité du travail ${ }^{3}$ et à vivre positivement l'incertitude que cela pourrait engendrer ${ }^{4}$. Étant donné ces changements, les jeunes sont dorénavant appelés à développer leur capacité à s'entreprendre de même qu'à acquérir un ensemble de compétences afin de mieux se préparer aux études, au travail ou à la vie en général ${ }^{5}$.

C'est pourquoi, depuis 2005, le ministère de l'Éducation et du Développement de la petite enfance du Nouveau-Brunswick développe des projets pédagogiques entrepreneuriaux (PPE) au sein des écoles francophones. Ces PPE s'insèrent dans le développement global des écoles communautaires du Nouveau-Brunswick qui ont pour vision que chaque enfant soit heureux de venir apprendre à l'école. Plus spécifiquement, la mission des écoles communautaires du Nouveau-Brunswick est de développer, dès le plus jeune âge, une culture de l'apprentissage autonome et de l'entrepreneuriat conscient au service de la santé globale des enfants, des familles, des partenaires et des communautés.

Lors d'un PPE, les jeunes sont amenés à jouer les rôles d'initiateurs, de réalisateurs et de gestionnaires dans le but de créer un produit, un service ou un événement qui répond à un besoin dans l'école ou la communauté. C'est alors que les jeunes de la maternelle à la $12^{\mathrm{e}}$ année découvrent et développent des qualités entrepreneuriales et des compétences essentielles à leur développement vie-carrière, tandis que les enseignants jouent le rôle de guide ou d'accompagnateur en intégrant au projet les résultats d'apprentissages de différents programmes d'études prescrits par la Province. Ainsi, il existe autant de projets entrepreneuriaux que de jeunes pour les entreprendre, par exemple: création et vente de livres, mise sur pied d'un service de collations santé, réalisation de vidéos pour contrer l'intimidation, semence et récolte d'un jardin communautaire, monter une galerie d'art, service de recyclage, concours de robotique, et bien d'autres.

Afin d'assurer le cheminement et la réussite des PPE au sein des écoles communautaires du Nouveau-Brunswick, l'organisme communautaire sans but lucratif Place aux compétences (PAC) est un acteur clé. En effet, sa mission est de collaborer à la mise en œuvre de programmes et de services novateurs au sein des écoles communautaires francophones axés sur la culture entrepreneuriale, la formation et le travail, et l'accueil d'élèves internationaux, en collaboration avec différents partenaires $^{6}$. Plus précisément, PAC vise à être un leadeur dans le développement de l'esprit d'entreprendre auprès des jeunes et des intervenants 
scolaires francophones tout en étant ouvert sur le monde ${ }^{5}$. De la sorte, PAC désire développer l'esprit d'entreprendre chez les jeunes francophones du Nouveau-Brunswick à travers la création, le soutien et le suivi de PPE qu'ils accompagnent financièrement à l'aide d'un fonds d'appui mis en place grâce aux contributions de divers partenaires publics et privés. Le fonds est accessible à tous les enseignants francophones de la maternelle à la $12^{\mathrm{e}}$ année du Nouveau-Brunswick qui désirent entreprendre un PPE.

Afin d'avoir la possibilité d'obtenir un financement pour la conduite d'un PPE, PAC a mis en place un mécanisme d'accompagnement de ces PPE à travers l'élaboration d'un scénario d'apprentissages intégrés (SAI) que le responsable d'un PPE doit compléter avant sa soumission. Dans ce cadre, les scénarios d'apprentissages intégrés sont des scénarios transdisciplinaires qui intègrent explicitement les résultats d'apprentissages des programmes d'études et les cibles éducatives retenues par le ministère de l'Éducation et du Développement de la petite enfance du Nouveau-Brunswick dans le cadre du développement de ses écoles communautaires. Le but d'un scénario d'apprentissages intégrés consiste à faire ressortir les liens entre divers types d'apprentissages dans différentes disciplines scolaires.

Après dix ans de mise en place, il est donc nécessaire de développer un cadre structurant stratégique afin de suivre la performance éducative et organisationnelle des PPE, et ce, dans une optique de support au développement de la capacité de s'entreprendre auprès des jeunes. En tant qu'organisme communautaire sans but lucratif, PAC est également soumis à certaines contraintes budgétaires et aux attentes des différents partenaires financiers du

\section{OBJECTIFS}

L'objectif principal de cet article est de proposer une plateforme structurante pour le suivi de limpact des PPE sur le développement de compétences, d'attitudes et de qualités entrepreneuriales chez les jeunes (voir tableau 1). Cet objectif s'inscrit dans une optique de gestion optimale de la qualité et par le besoin de développer un cadre structurant assurant le développement de la compétence fonds d'appui. Dès lors, il est primordial de mettre en place des outils d'évaluation continue afin d'allouer efficacement les différentes ressources dans le but d'atteindre les objectifs éducatifs visés et de permettre aux intervenants de mettre en place leur PPE dans les meilleures conditions.

C'est alors que le développement d'un cadre structurel stratégique clair permettrait d'évaluer de manière continue les composantes de PAC afin de déterminer les éléments qui fonctionnent et ceux qui devraient être améliorés. En effet, il est essentiel d'évaluer annuellement si les objectifs théoriques initiaux coïncident avec les réalités du terrain. De cette manière, PAC opérationnaliserait ses objectifs à travers la création d'un cadre structurant correspondant aux besoins du terrain : besoins des écoles, des intervenants et des élèves afin de rendre les élèves réellement initiateurs, réalisateurs et gestionnaires des PPE. Dès lors, à partir d'une telle approche d'opérationnalisation, PAC serait en mesure de mieux accompagner les PPE à travers une meilleure allocation des ressources grâce à la mise en place d'un mécanisme de collecte de données de haute qualité, fiables et exploitables. L'opérationnalisation des résultats se ferait à l'aide de statistiques descriptives pour suivre les tendances globales des PPE à long terme.

Il s'avère donc que la construction d'une stratégie à long terme n'est possible qu'en impliquant les intervenants de terrain et en créant des liens pérennes avec le milieu de la recherche afin de développer l'utilisation de la plateforme structurante. Dans ce contexte, la question de recherche centrale est la suivante : quels sont les mécanismes à mettre en place pour soutenir les PPE et maximiser leur impact sur la stimulation de l'esprit d'entreprendre chez les jeunes?

«s'entreprendre » par le biais d'une approche expérientielle.

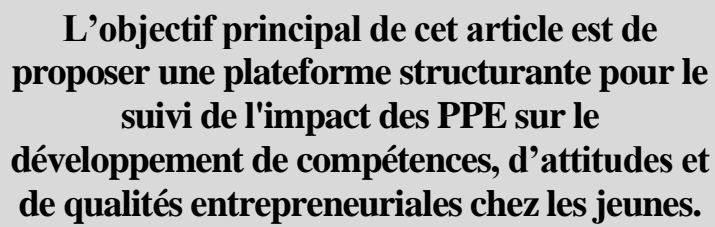

L'objectif principal de cet article est de proposer une plateforme structurante pour le suivi de l'impact des PPE sur le développement de compétences, d'attitudes et de qualités entrepreneuriales chez les jeunes. 
Tableau 1 - Compétence, qualités et attitudes entrepreneuriales visées par les écoles communautaires du Nouveau-Brunswick

\begin{tabular}{|c|c|c|}
\hline COMPÉTENCE & QUALITÉS & ATTITUDES \\
\hline \multirow[t]{6}{*}{$\begin{array}{l}\text { S'entreprendre : } \\
\text { Se sentir compétent à } \\
\text { entreprendre sa vie, à prendre } \\
\text { des décisions et à poser les } \\
\text { actions nécessaires pour } \\
\text { réaliser la vie que l'on désire. }\end{array}$} & $\begin{array}{l}\text { Leadership : } \\
\text { Influencer, donner goût aux autres } \\
\text { de se joindre avec énergie au projet } \\
\text { proposé. }\end{array}$ & $\begin{array}{l}\text { Fierté identitaire et } \\
\text { culturelle : } \\
\text { Capacité de s'affirmer avec } \\
\text { assurance en recourant à sa } \\
\text { langue et à sa culture dans la } \\
\text { sphère privée comme dans la } \\
\text { sphère publique. }\end{array}$ \\
\hline & Esprit d’équipe : & Recherche constante \\
\hline & $\begin{array}{l}\text { Travailler ensemble dans la } \\
\text { poursuite d'un but commun. }\end{array}$ & $\begin{array}{l}\text { d'innovation : } \\
\text { Habitude régulière de « sortir } \\
\text { du carré », de chercher } \\
\text { plusieurs solutions devant une } \\
\text { situation donnée. }\end{array}$ \\
\hline & $\begin{array}{l}\text { Confiance en soi : } \\
\text { Se sentir capable de faire quelque } \\
\text { chose ou ce qu'il y a à faire. }\end{array}$ & $\begin{array}{l}\text { Engagement } \\
\text { communautaire : Action de } \\
\text { s'impliquer, dans un esprit } \\
\text { d'entraide et de générosité, } \\
\text { dans l'amélioration du } \\
\text { potentiel ou des conditions de } \\
\text { vie de divers membres de la } \\
\text { communauté en réponse à des } \\
\text { besoins spécifiques. }\end{array}$ \\
\hline & $\begin{array}{l}\text { Sens des responsabilités : Faire ce } \\
\text { qui doit être fait et en assumer les } \\
\text { conséquences. Réaliser les mandats } \\
\text { confiés. }\end{array}$ & \\
\hline & $\begin{array}{l}\text { Sens de l'organisation : } \\
\text { Euvrer avec méthode, efficacité et } \\
\text { efficience. }\end{array}$ & \\
\hline
\end{tabular}

\section{MÉTHODOLOGIE}

\section{La méthodologie employée repose sur une démarche quali-quantitative. [...] utilise un processus d'observation participative dans un contexte de recherche-action impliquant les utilisateurs des connaissances dans une dynamique collaborative.}

La méthodologie employée repose sur une démarche quali-quantitative. Plus spécifiquement, elle utilise un processus d'observation participative dans un contexte de recherche-action impliquant les utilisateurs des connaissances dans une dynamique collaborative. La méthodologie implique ainsi chercheurs, représentants de PAC, acteurs du terrain (enseignants, agents communautaires et coordonnateurs des écoles communautaires) et élèves. En outre, l'accord du ministère de l'Éducation et du Développement de la petite enfance du NouveauBrunswick et l'accord des directeurs et des directrices des districts scolaires francophones de la province du Nouveau-Brunswick ont été obtenus. Une demande d'approbation éthique a également été soumise au Comité d'éthique de la recherche avec les êtres humains de l'Université de Moncton. L'approbation de ce comité a été obtenue (dossier no 1415-026). 


\section{RÉSULTATS}

\section{L'objectif est de proposer à PAC des outils facilement utilisables afin qu'ils puissent mener de manière intégrée et autonome l'évaluation continue de ces projets tout en recevant, au besoin, l'appui collaboratif de partenaires externes.}

Dans le cadre de cette recherche participative action, divers outils de collecte et de gestion de données concernant les PPE ont été mis en place. Il s'agit d'éléments stratégiques qui sont à la base d'une plateforme structurante visant l'évaluation continue, le suivi et le soutien des PPE ainsi que l'amélioration de leur impact auprès des jeunes, notamment en matière de la compétence «s'entreprendre ». En d'autres mots, l'objectif est de proposer à PAC des outils facilement utilisables afin qu'ils puissent mener de manière intégrée et autonome l'évaluation continue de ces projets tout en recevant, au besoin, l'appui collaboratif de partenaires externes.

\subsection{Outils de collecte de données}

Dans le but de mesurer les dimensions centrales de l'apprentissage de la compétence «s'entreprendre » ainsi que des qualités et des attitudes entrepreneuriales, trois outils de collecte de données ont été développés : le questionnaire ComQuAtt ${ }^{\circledR}$, une base de données pour le suivi des PPE et une banque de données globale de gestion des PPE. Le premier permet de représenter les indicateurs de performance, le second est un outil de suivi des PPE à long terme et le troisième permet d'effectuer une collecte de données pour avoir la possibilité de réaliser des statistiques de monitorage tout en permettant la traçabilité de l'information recueillie.

\subsection{Questionnaire ComQuAtt®}

D'emblée, des indicateurs clés cherchant à évaluer les compétences, les qualités ainsi que les attitudes entrepreneuriales des jeunes, tel que décrit au tableau 1, ont été ciblés. Ces indicateurs ont servi de base afin de concevoir un questionnaire évaluatif cherchant à mesurer les retombées des PPE sur l'esprit d'entreprendre des jeunes. Plus spécifiquement, le questionnaire comprend tout d'abord diverses questions sociodémographiques permettant de situer chaque répondant vis-à-vis de son PPE et de ses caractéristiques individuelles. Il comprend ensuite divers groupes d'items se rapportant aux dimensions étudiées, c'est-à-dire à la compétence «s'entreprendre», aux qualités (leadership, esprit d'équipe, confiance en soi, sens des responsabilités et sens de l'organisation) et aux attitudes (fierté identitaire et culturelle, recherche constante d'innovation et engagement communautaire) entrepreneuriales (voir tableau 1). Tous ces items sont mesurés à l'aide d'échelles de type Likert. Une question qualitative est présentée à la fin du questionnaire afin de permettre à chaque jeune de s'exprimer en quelques mots sur son expérience personnelle au sein du PPE auquel il a participé. Ce questionnaire, qui a démontré avoir de bonnes propriétés psychométriques, permettra à PAC d'effectuer un suivi avant et après la mise en place des PPE au sein des écoles communautaires.

\subsection{Base de données pour le suivi des PPE}

En plus d'avoir élaboré un questionnaire évaluatif, cette recherche participative action a également permis de développer une base de données Excel regroupant les données les plus pertinentes des scénarios d'apprentissages intégrés (données avant-projet) et des rapports finaux (données après projet) que doivent remplir les responsables de chaque PPE. Ces données permettent d'avoir une fiche technique détaillée de chaque projet grâce à un accès aisé et rapide aux différentes données. Cette base de données Excel permettra à $\mathrm{PAC}$ d'améliorer la structure de ces deux documents à compléter afin de récolter de manière efficace les informations nécessaires à l'évaluation continue des PPE. Cette base modulable selon les besoins de PAC et les caractéristiques des PPE pourra donc évoluer suivant les besoins liés à la poursuite d'une évaluation continue efficace des PPE.

Chaque PPE est référencé selon un code spécifique au sein de cette base de données Excel et les données inscrites sur la ligne correspondante permettent d'établir une carte d'identité 
détaillée de chaque projet. Entre autres, la base permet de déterminer la fiche technique détaillée du projet (le nom du PPE, l'école, le district, etc.) ainsi que ses caractéristiques (type de PPE, nombre d'élèves, niveaux scolaires concernés). Il permet également d'établir qui sont les intervenants scolaires liés aux projets (enseignant, agent communautaire, direction, coordonnateur des écoles communautaires, etc.) ainsi que les partenaires communautaires impliqués. La base de données permet aussi de répertorier si le projet a bénéficié d'une aide financière de PAC et si les responsables du projet ont rendu un rapport suite à la réalisation du projet comme attendu.

Au-delà des données techniques liées à la mise en place du projet, les objectifs pédagogiques et éducatifs visés dans le cadre de sa réalisation sont également encodés. Chaque responsable de PPE doit indiquer dans le scénario d'apprentissages intégrés les objectifs visés par le PPE en termes d'apprentissages tels que les liens avec les programmes d'études, les qualités entrepreneuriales visées, les habiletés développées en lien avec les technologies de l'information et de la communication, les secteurs de travail associés et les compétences vie-carrière qui seront développées au cours du projet. Il est à noter que ces

\section{CONCLUSION}

L'objectif principal de cet article était de proposer une plateforme structurante pour le suivi de l'impact des projets pédagogiques entrepreneuriaux (PPE) sur le développement de certaines compétences, attitudes et qualités entrepreneuriales déterminées chez les jeunes dans une optique de gestion optimale à long terme (voir tableau 1). Dans l'ensemble, les résultats démontrent la faisabilité de cette plateforme structurante qui comprend divers outils de suivi continu des PPE dont un questionnaire d'évaluation, une base de données pour le suivi des PPE et une banque de données globale de gestion des PPE. Grâce à de tels outils de suivi continu, l'organisme communautaire Place aux compétences (PAC) est mieux outillé afin d'évaluer les retombées de ces PPE sur l'esprit d'entreprendre des jeunes au sein de leur communauté. informations sont des objectifs d'avant-projet et ne permettent donc pas d'évaluer les apprentissages réellement développés dans le cadre du PPE. Enfin, les démarches pour promouvoir le marketing éducatif du PPE sont également répertoriées à partir des informations indiquées dans les deux documents.

\subsection{Banque de données globale de gestion des PPE}

Enfin, une banque de données SPSS a été créée dans le but de rassembler l'ensemble des données récoltées en un seul lieu, permettant ainsi une meilleure traçabilité de l'information et un suivi continu des PPE à long terme. Ces données permettront également de réaliser des statistiques de monitorage. Il s'agit en fait d'un croisement entre les données colligées par l'entremise du questionnaire évaluatif et des divers renseignements que l'on retrouve à l'intérieur des scénarios d'apprentissages intégrés et des rapports finaux. En effet, cet outil permet de suivre les PPE dans le cadre de leur évaluation continue (participation au sondage, nombre de sondages complétés reçus par l'équipe de recherche) en lien avec les données colligées dans la base de données Excel.

Cependant, il est clair que la mise en application de ces divers outils pourrait susciter des défis d'ordre opérationnel. En effet, l'implantation ou le fonctionnement d'une telle plateforme structurante d'évaluation continue pourrait susciter des coûts importants se traduisant par l'ajout d'une ou de plusieurs ressources humaines, par exemple. À cela s'ajoute la capacité d'exploitation d'une telle plateforme et encore la qualité des données colligées. Dès lors, l'amélioration continue des PPE, avec pour objectif d'améliorer leur impact, est tributaire d'une approche collaborative laissant place à la flexibilité, à l'adaptation et à l'innovation locale. C'est donc par la sensibilisation, la formation et l'accompagnement des acteurs clés des milieux scolaires et communautaires que la réalisation d'expériences entrepreneuriales à l'école, dont leur évaluation, sera facilitée. 
Il s'avère important de souligner la nécessité de créer des partenariats entre les organismes communautaires et les centres de recherche, par exemple les universités, afin de répondre aux besoins du terrain en matière d'outils et de recommandations pratiques et exploitables. En fait, grâce à l'approche collaborative et entrepreneuriale des écoles francophones du Nouveau-Brunswick, les PPE vont continuer à rayonner dans les écoles communautaires francophones du Nouveau-Brunswick et vont évoluer selon les mécanismes structurants de l'évaluation continue réalisée par PAC.

Il s'avère important de souligner la nécessité de créer des partenariats entre les organismes communautaires et les centres de recherche, par exemple les universités, afin de répondre aux besoins du terrain en matière d'outils et de recommandations pratiques et exploitables.

En cherchant à poursuivre les recherches de manière systématique sur cette thématique, il pourrait être pertinent d'approfondir la démarche structurante proposée en y incorporant une sphère plus qualitative où des entretiens avec les utilisateurs de la plateforme, les jeunes ayant participé aux PPE ou les responsables des PPE pourraient apporter leurs points de vue sur les retombées en termes d'acquis ou encore en termes de pistes à améliorer. De cette manière, un suivi de type longitudinal utilisant cette plateforme structurante pourrait également s'avérer propice afin d'établir un tracé des acquis en termes de retombées entrepreneuriales auprès des jeunes.

Dans le cadre d'un processus d'évaluation continue, l'amélioration du processus passe également par la formation des (futurs) responsables de PPE afin qu'ils maîtrisent les différents outils pour développer efficacement un PPE en respectant les objectifs et valeurs sous-jacents au développement de la compétence «s'entreprendre ». Un tel module devrait rappeler les objectifs stratégiques des PPE en matière de s'entreprendre et inclure une trousse d'outils pour aider les demandeurs de PPE à cibler les apprentissages attendus.
En bref, les PPE sont une occasion majeure d'acquisition de la compétence «s'entreprendre» ainsi que des qualités et attitudes entrepreneuriales sous-jacentes. Les PPE sont donc porteurs d'un haut potentiel de développement chez les jeunes et d'une préparation pour le monde du travail. À cet égard, il s'avère important que l'évaluation continue des PPE et de leur impact soit une approche stratégique majeure pour l'optimisation de l'investissement accordé aux PPE

\section{BIBLIOGRAPHIE ET NOTES}

${ }^{1}$ Clara Jates, économiste et enseignante de formation, est passionnée par l'éducation entrepreneuriale. Sa passion l'a amenée à travailler au Nouveau-Brunswick avec Place aux compétences et l'Université de Moncton sur l'évaluation des projets pédagogiques entrepreneuriaux. De retour en Belgique, elle coordonne désormais les Mini-Entreprises de la province du Hainaut pour l'association sans but lucratif Les Jeunes Entreprises (LJE).

${ }^{2}$ Pepin, M. (2013). Les fondements éducatifs de l'entrepreneuriat scolaire au primaire et au secondaire, dans G. Samson (sous la direction de), Les retombées de l'entrepreneuriat éducatif, Presses de l'Université du Québec, p. 169-189.

${ }^{3}$ Uy, Marilyn A., Chan, Kim-Yin, Sam, Yoke Loo, Ho, Moon-ho Ringo, Chernyshenko, Oleksandr S. (2015). « Proactivity, adaptability and boundaryless career attitudes: The mediating role of entrepreneurial alertness », Journal of Vocational Behavior, vol. 11, p. $115-123$.

${ }^{4}$ Trevor-Roberts, Edwin (2006). « Are you sure? The role of uncertainty in career », Journal of Employment Counseling, vol. 43, no 3, p. 98-116.

${ }^{5}$ Ministère de l'Éducation et du Développement de la petite enfance du Nouveau-Brunswick (MEDPE, 2014). Liens entre le développement vie-carrière et l'esprit d'entreprendre dans les écoles communautaires entrepreneuriales. Récupéré le 20 avril 2015 de : http://www.gnb.ca/0000/publications/Comm EcoleFran/ECENB\%20Liens\%20viecarriere\%20et\%20esprit\%20dentreprendre.pdf ${ }^{6}$ Place aux compétences (2015). Mission et vision. Récupéré le 26 avril 2015 de : http://www.pacnb.org/ index.php/fr/a-propos/mission-et-vision

${ }^{7}$ Ministère de l'Éducation et du Développement de la petite enfance du Nouveau-Brunswick (MEDPE, 2011). Profil de sortie - Définitions. Récupéré le 20 avril $2015 \mathrm{de}:$ http://www.gnb.ca/0000/publications/ CommEcoleFran/ECNB Profil de sortie-d\%C3\%A9 finitions.pdf 